\title{
Tumores de cães e gatos diagnosticados no semiárido da Paraíba ${ }^{1}$
}

\author{
Rachel L.F.S. Andrade ${ }^{2}$, Diego M. Oliveira², Antônio Flávio M. Dantas ${ }^{2 *}$, Almir Pereira de Souza ${ }^{2}$, \\ Pedro Isidro da Nóbrega Neto $^{2}$ e Franklin Riet-Correa ${ }^{2}$
}

\begin{abstract}
Andrade R.L.F.S., Oliveira D.M., Dantas A.F.M., Souza A.P., Nóbrega Neto P.I. \& Riet-Correa F. 2012. [Tumors in dogs and cats diagnosed in semiarid of Paraíba, Brazil.] Tumores de cães e gatos diagnosticados no semiárido da Paraíba. Pesquisa Veterinária Brasileira 32(10):1037-1040. Hospital Veterinário, CSTR, Universidade Federal de Campina Grande, Cx. Postal 64, Patos, PB 58700-970, Brazil. E-mail: dantas.af@uol.com.br

This paper reports the frequency of tumors diagnosed in dogs and cats at the Veterinary Hospital of the Federal University of Campina Grande in Patos, northeastern Brazil, between 2003 and 2010. All necropsy and biopsy protocols from the Pathology Laboratory were revised and data related to the tumors were analyzed. In dogs, tumors of the skin and anexa were the most frequently diagnosed (46.7\%), followed by tumors of the mammary gland $(24 \%)$, genital system $(10,3 \%)$, and alimentary system (6.5\%). Malignant tumors $(78 \%)$ were more frequent than bening tumors $(22 \%)(p=0.001)$. In cats the frequency of skin and mammary gland tumors was the same (39.4\%), followed by tumors of the digestive system (8.5\%) and liver (5.7\%). In cats, $95.8 \%$ of the tumors were malignant. Due to the diversity of the tumors observed in dogs and cats it is difficult for the practitioner to diagnose and treat tumors in these species. As a result, the systematic histologic diagnosis and the determination of the epidemiology of the tumors, in the different regions, are necessary to decrease tumor mortality.
\end{abstract}

INDEX TERMS: Tumors, canine, feline, histophatology, oncology.

RESUMO.- Objetivou-se com este estudo determinar a frequência dos tumores diagnosticados em cães e gatos no Hospital Veterinário da Universidade Federal de Campina Grande (UFCG), Patos, Paraíba, Brasil, entre os anos de 2003 a 2010. Foram revisados todos os protocolos de necropsias e biópsias realizadas no Laboratório de Patologia Animal da referida instituição, e computados os dados referentes aos animais afetados. Os tumores mais frequentes em cães ocorreram na pele e anexos $(46,7 \%)$, seguido da glândula mamária (24\%), sistema genital $(10,3 \%)$ e sistema digestório $(6,5 \%)$. Os tumores malignos foram mais frequentes que os benignos $(\mathrm{p}=0,001)$, representando $78 \%$ e $22 \%$ respectivamente. Em gatos, as frequências de neoplasmas de pele e glândula mamária foram idênticas

\footnotetext{
${ }^{1}$ Recebido em 2 de maio de 2012.

Aceito para publicação em 12 de julho de 2012.

Parte da Dissertação de Mestrado do primeiro autor, Programa de Pós-Graduação em Medicina Veterinária, Universidade Federal de Campina Grande (UFCG), Av. Universitária s/n, Bairro Santa Cecília, Patos, PB 58708-110, Brasil.

${ }^{2}$ Hospital Veterinário, Centro de Saúde e Tecnologia Rural (CSTR), UFCG, Cx. Postal 64, Patos, PB 58700-970. *Autor para correspondência: dantas.af@uol.com.br
}

$(39,4 \%$ cada), seguidas das do sistema digestório $(8,5 \%)$ e fígado $(5,7 \%)$. Os tumores malignos representaram $95,8 \%$ dos tumores diagnosticados nesta espécie. Cães sem raça definida tiveram menor frequência $(16 \%)$ de tumores do que animais de diferentes raças $(37,2 \%)(p<0,0001)$. A ampla variedade de neoplasmas que acomete cães e gatos dificulta ao veterinário o diagnóstico clínico e o tratamento das mesmas, sendo recomendável o diagnóstico histológico sistemático de todas as lesões suspeitas de serem neoplasias. Além disso, são necessários estudos que determinem os fatores epidemiológicos envolvidos no desenvolvimento dos tumores que acometem os cães e gatos, nas diferentes regiões, a fim de serem tomadas medidas para diminuir sua ocorrência e letalidade.

TERMOS DE INDEXAÇÃO: Tumores, cães, gatos, histopatologia, oncologia.

\section{INTRODUÇÃO}

As neoplasias estão entre as principais causas de morte em cães e gatos (Meuten 2002, Daleck et al. 2008, Fighera et al. 2008). Os diversos tipos de neoplasias observadas em pequenos animais podem variar geograficamente devido 
às diferenças de susceptibilidade e hábitos dos animais e aos fatores ambientais (Souza 2005), sendo importante o desenvolvimento de estudos locais e regionais que permitam fornecer informações acerca das principais desordens neoplásicas nas diferentes regiões.

Muitos tumores são diagnosticados anualmente pelo Laboratório de Patologia Animal (LPA) do Hospital Veterinário da Universidade Federal de Campina Grande (UFCG), Patos, Paraíba, Brasil. Grande parte desses tumores é maligna e, muitas vezes, é a causa da morte ou a razão para eutanásia devido ao prognóstico desfavorável e às complicações sistêmicas. 0 conhecimento da frequência, assim como as características epidemiológicas são importantes para o tratamento e profilaxia dos tumores. 0 objetivo deste trabalho foi determinar a frequência e algumas características epidemiológicas dos tumores diagnosticadas em cães e gatos no LPA durante o período de 2003 a 2010.

\section{MATERIAL E MÉTODOS}

Foram revisados todos os protocolos de necropsias e biópsias realizadas em cães e gatos no LPA, entre os anos de 2003 a 2010. Das fichas dos animais que apresentaram afecções neoplásicas foram obtidos os dados referentes à espécie, sexo, idade, raça, localização anatômica dos tumores e classificação morfológica. Quando necessário, novas lâminas foram preparadas a partir dos arquivos de blocos de parafina.

Os tumores foram classificados em grupos, de acordo com a classificação utilizada por Meuten (2002), que é similar à classificação histológica preconizada pela Organização Mundial da Saúde e pelo Armed Forces Institute of Pathology (OMS e AFIP) (Misdorp et al. 1999). Os tumores foram classificados ainda segundo o sistema afetado, a faixa etária, o sexo e a raça do animal acometido. As faixas etárias foram consideradas da seguinte forma: jovens (até um ano de idade), adultos (de um a nove anos de idade) e idosos (acima de nove anos de idade), de acordo com limites anteriormente estipulados (Fighera et al. 2008, Oliveira Filho et al. 2010). Quanto ao sexo, os animais foram classificados como machos e fêmeas, independente de serem castrados ou não. As raças foram computadas conforme descrito em cada protocolo.

Foi calculada a frequência de cada tipo histológico em relação ao total de tumores diagnosticados, a faixa etária, a localização anatômica e as características morfológicas de acordo com a origem celular e comportamento biológico. Para a análise estatística foi realizado o teste de Qui-Quadrado, utilizando o programa BioEstat 5.0.

\section{RESULTADOS}

Durante o período de 2003 a 2010, foram recebidos 1.272 espécimes de biópsia (405 [38\%]) ou cadáveres (867 [68\%]) de cães e gatos no LPA. Desses, 945 (74\%) eram cães e 327 (26\%) eram gatos. Os tumores representaram $27 \%(344 / 1.272)$ do total de diagnósticos, acometendo mais cães (275/945; [29\%]) do que gatos (69/327; [21\%]) $(\mathrm{p}=0,0366)$. Dos animais submetidos à necropsia, $94(11 \%)$ morreram ou foram eutanasiados em decorrência de processos neoplásicos, e entre as biópsias, 250 (62\%) animais apresentaram tumores.

Do total de animais (1.272), $652(51,3 \%)$ eram machos, $581(45,7 \%)$ eram fêmeas e 39 (3\%) não tiveram o sexo informado em seus protocolos. A frequência de tumores em fêmeas foi de $39,9 \%$ e em machos $21,2 \%$, sendo as fê- meas mais acometidas por tumores $(\mathrm{p}=0,0003)$, tanto para os cães ( $p=0,0491)$ quanto para gatos $(p<0,0001)$. Em relação à idade, os cães acometidos por processos neoplásicos tinham entre quatro meses e 17 anos de idade, enquanto os gatos apresentaram idade entre 1 e 20 anos, com idade média de 7,3 anos para os cães e 8,8 anos para os gatos. Os tumores foram mais frequentes em animais idosos, tanto em cães quanto em gatos ( $p<0,0001)$, como observado no Quadro 1. Os cães sem raça definida foram menos frequentemente afetados $(55 / 429 ;[12,8 \%])$ por tumores do que os cães de raça definida $(157 / 453 ;[34,6 \%])(p<0,0001)$. Não foi observada diferença significativa entre gatos de diferentes raças. No Quadro 2 observa-se a frequência de tumores em cães e gatos distribuídas nas diferentes raças.

Foi observado que $200(58,1 \%)$ animais apresentavam um único tumor, enquanto que tumores múltiplos foram observados em 144 (41,9\%); destes, 20,1\% (29/144) eram metastáticos e 11,8\% (17/144) eram tumores com tipos histológicos distintos. Este último fator fez com que o nú-
Quadro 1. Total de tumores diagnosticados no LPA da UFCG, Patos, Paraíba, distribuído pela idade de cães e gatos, durante o período de 2003 a 2010

\begin{tabular}{lccccc}
\hline & \multicolumn{2}{c}{ Cães } & & \multicolumn{2}{c}{ Gatos } \\
\cline { 2 - 3 } \cline { 5 - 6 } & $\begin{array}{c}\text { Total de } \\
\text { amostras } \\
\text { recebidas }\end{array}$ & $\begin{array}{c}\text { Total de } \\
\text { neoplasias }\end{array}$ & & $\begin{array}{c}\text { Total de } \\
\text { amostras } \\
\text { recebidas }\end{array}$ & $\begin{array}{c}\text { Total de } \\
\text { neoplasias }\end{array}$ \\
\hline Até 1 ano (jovens) & 219 & $8(3,6 \%)$ & & 77 & $1(1,3 \%)$ \\
$1-9$ anos (adultos) & 483 & $166(34,4 \%)$ & & 158 & $31(19,6 \%)$ \\
$>9$ anos (idosos) & 116 & $75(64,7 \%)$ & & 57 & $35(61,4 \%)$ \\
NI* & 127 & $26(20,5 \%)$ & & 35 & $2(5,7 \%)$ \\
TOTAL & 945 & $275(29 \%)$ & & 327 & $69(21 \%)$
\end{tabular}

* Não informado.

Quadro 2. Total de tumores diagnosticados no LPA da UFCG, Patos, Paraíba, distribuído por raças de cães e gatos durante o período de 2003 a 2010

\begin{tabular}{|c|c|c|c|c|c|}
\hline Cães & $\begin{array}{c}\text { Total de } \\
\text { amostras } \\
\text { recebidas }\end{array}$ & $\begin{array}{l}\text { Total de } \\
\text { tumores }\end{array}$ & Gatos & $\begin{array}{c}\text { Total de } \\
\text { amostras } \\
\text { recebidas }\end{array}$ & $\begin{array}{l}\text { Total de } \\
\text { tumores }\end{array}$ \\
\hline $\mathrm{SRD}^{\mathrm{a}}$ & 429 & $55(12,8 \%)$ & $\mathrm{SRD}^{\mathrm{a}}$ & 246 & $53(21,5 \%)$ \\
\hline Poodle & 103 & $31(30,1 \%)$ & Siamês & 38 & $11(28,9 \%)$ \\
\hline Pinscher & 83 & $26(31,3 \%)$ & Angorá & 2 & $2(100 \%)$ \\
\hline Rottweiler & 50 & $23(46 \%)$ & Persa & 8 & $1(12,5 \%)$ \\
\hline Pit Bull & 56 & $19(33,9 \%)$ & $\mathrm{NI}^{\mathrm{b}}$ & 33 & $2(6,1 \%)$ \\
\hline Dobermann & 18 & $11(61,1 \%)$ & TOTAL & 327 & $69(21,1 \%)$ \\
\hline Pastor Alemão & 31 & $11(35,5 \%)$ & & & \\
\hline Cocker Spaniel & 41 & $9(21,9 \%)$ & & & \\
\hline Dogue Alemão & 11 & $8(72,7 \%)$ & & & \\
\hline Pequinês & 8 & $4(50 \%)$ & & & \\
\hline Fila Brasileiro & 11 & $3(27,3 \%)$ & & & \\
\hline Dachshund & 7 & $3(42,8 \%)$ & & & \\
\hline Boxer & 4 & $3(75 \%)$ & & & \\
\hline Pointer Inglês & 5 & $1(20 \%)$ & & & \\
\hline Weimaraner & 4 & $1(25 \%)$ & & & \\
\hline Dálmata & 5 & $1(20 \%)$ & & & \\
\hline Beagle & 2 & $1(50 \%)$ & & & \\
\hline Husky Siberiano & 2 & $1(50 \%)$ & & & \\
\hline Yorkshire & 1 & $1(100 \%)$ & & & \\
\hline Outras raças & 11 & $1(100 \%)$ & & & \\
\hline $\mathrm{NI}^{\mathrm{b}}$ & 63 & $63(100 \%)$ & & & \\
\hline TOTAL & 945 & $275(29,1 \%)$ & & & \\
\hline
\end{tabular}

${ }^{\mathrm{a}}$ Sem raça definida, ${ }^{\mathrm{b}}$ não informada. 
mero de diagnósticos fosse superior ao número de animais avaliados. Desta forma, houve 291 diagnósticos de tumores em cães e 71 em gatos.

De acordo com o sistema afetado, os tumores mais frequentes em cães ocorreram na pele e anexos (46,7\%), seguidas da glândula mamária (24\%), sistema genital $(10,3 \%)$ e sistema digestório $(6,5 \%)$. Os tumores malignos foram mais frequentes que os benignos $(p=0,001)$, representando $78 \%$ e $22 \%$ respectivamente. Em gatos, a frequência de tumores de pele e glândula mamária fora idêntica $(39,4 \%$ cada), seguida do sistema digestivo $(8,5 \%)$ e fígado $(5,7 \%)$. Como nos cães, os gatos apresentaram maior número de tumores malignos $(95,8 \%)$ em relação aos benignos $(4,2 \%)$. Do total de tumores observados, $50,3 \%$ foram epiteliais e $49,7 \%$ mesenquimais.

Os tumores diagnosticados, de acordo com o sistema, estão listados no Quadro 3. Os tumores de pele mais observados em cães foram: carcinoma de células escamosas
(CCE), hemangiossarcoma, mastocitoma e histiocitoma. Em gatos, o CCE foi o mais frequente entre os tumores de pele. Tanto em cães quanto em gatos, tumores malignos foram os mais observados na glândula mamária. No sistema genital, o tumor venéreo transmissível (TVT) foi mais frequente, representando $50 \%(15 / 30)$ de todos os tumores observados nesse sistema. Os tumores da cavidade oral foram os mais frequentes no sistema digestório, representando $75 \%$ $(15 / 20)$ de todos os tumores do trato digestivo nos cães e $66,7 \%(4 / 6)$ nos gatos.

No fígado, colangiocarcinomas foram os mais diagnosticados. Destes, três casos foram observados em gatas adultas, entre 8 e 20 anos, sem raça definida, e estavam associados à infecção pelo trematódeo Platynosomum fastosum.

\section{DISCUSSÃO}

Este estudo demonstrou que a frequência de tumores em cães e gatos na região do semiárido paraibano é alta, prin-

Quadro 3. Frequência dos tumores diagnosticados em cães e gatos no LPA da UFCG, Patos/PB, Brasil, durante o período de 2003 a 2010, distribuída segundo a classificação histopatológica e o sistema afetado

\begin{tabular}{|c|c|c|c|c|c|c|c|}
\hline Sistema & $\begin{array}{c}\text { Classificação } \\
\text { histopatológica }\end{array}$ & Cães & Gatos & Sistema & $\begin{array}{c}\text { Classificação } \\
\text { histopatológica }\end{array}$ & Cães & Satos \\
\hline \multirow[t]{23}{*}{ PELE E ANEXOS } & & 136 & 28 & DIGESTÓRIO & & 19 & 6 \\
\hline & $\mathrm{CCE}^{\mathrm{a}}$ & 19 & 20 & & Epúlide fibromatoso & 5 & \\
\hline & Hemangiossarcoma & 19 & 3 & & Fibrossarcoma & 3 & 1 \\
\hline & Mastocitoma & 18 & & & $\mathrm{CCE}^{\mathrm{a}}$ & 2 & 2 \\
\hline & Histiocitoma & 10 & 1 & & Melanoma & 2 & \\
\hline & Lipoma & 9 & & & Papiloma & 2 & \\
\hline & Melanoma & 8 & & & $\mathrm{Ca}^{\mathrm{b}}$ salivar & 2 & \\
\hline & TVT $^{\mathrm{c}}$ & 7 & & & Fibroma & 1 & 1 \\
\hline & $\mathrm{Ca}^{\mathrm{b}}$ de gl. perianal & 6 & & & Ameloblastoma & 1 & \\
\hline & Adenoma de gl. perianal & 5 & & & $\mathrm{Ca}^{\mathrm{b}}$ duodenal & & 1 \\
\hline & $\mathrm{Ca}^{\mathrm{b}}$ de céls. basais & 5 & 2 & & $\mathrm{Ca}^{\mathrm{b}}$ gástrico & & 1 \\
\hline & Fibrossarcoma & 5 & 1 & & $\mathrm{Ca}^{\mathrm{b}}$ pancreático & 1 & \\
\hline & Linfoma & 4 & & HEMOLINFÁTICO & & 15 & 1 \\
\hline & Adenoma sebáceo & 3 & & & Linfoma & 14 & 1 \\
\hline & Hemangioma & 3 & & & Hemangiossarcoma & 1 & \\
\hline & Papiloma & 3 & & NERVOSO & & 3 & \\
\hline & Tricoblastoma & 3 & & & Quimiodectoma & 1 & \\
\hline & $\mathrm{Ca}^{\mathrm{b}}$ sebáceo & 2 & & & Oligodendroglioma & 1 & \\
\hline & Fibroma & 2 & & & Meningioma & 1 & \\
\hline & Hemangiopericitoma & 2 & & MUSCULAR & & 2 & \\
\hline & Adenoma meibomiano & 1 & & & Rabdomiossarcoma & 2 & \\
\hline & Lipossarcoma & 1 & & FÍGADO & & 3 & 4 \\
\hline & Plasmocitoma & 1 & 1 & & Colangiocarcinoma & 2 & 3 \\
\hline \multicolumn{2}{|c|}{ GLÂNDULA MAMÁRIA } & 70 & 28 & & $\mathrm{Ca}^{\mathrm{b}}$ hepatocelular & & 1 \\
\hline & Carcinossarcoma & 27 & & & Osteossarcoma extra-esquelético & o 1 & \\
\hline & $\mathrm{Ca}^{\mathrm{b}}$ simples & 23 & 27 & ENDÓCRINO & & & \\
\hline & $\mathrm{Ca}^{\mathrm{b}}$ complexo & 12 & & & Feocromocitoma & 1 & \\
\hline & Adenoma simples & 5 & 1 & OCULAR & & & \\
\hline & Tumor misto benigno & 3 & & & TVT $^{\mathrm{c}}$ & 3 & \\
\hline \multicolumn{2}{|c|}{ SISTEMA GENITAL FEMININO } & 30 & 2 & ÓSSEO & & & \\
\hline & TVT $^{c}$ & 9 & & & Osteossarcoma & 6 & \\
\hline & Leiomioma & 1 & 1 & RESPIRATÓRIO & & 3 & \\
\hline & Luteoma & & 1 & & $\mathrm{Ca}^{\mathrm{b}}$ bronquio-alveolar & 1 & \\
\hline & Tumor de céls. da granulosa & 1 & 1 & & $\mathrm{Ca}^{\mathrm{b}}$ nasal & 1 & \\
\hline \multirow[t]{6}{*}{ MASCULINO } & TVT $^{c}$ & 6 & & & $\mathrm{CCE}^{\mathrm{a}}$ & 1 & \\
\hline & Seminoma & 4 & & & & & \\
\hline & Sertolioma & 4 & & & & & \\
\hline & Ca prostático & 2 & & & & & \\
\hline & Tumor de céls. de Leydig & 2 & & & & & \\
\hline & Hemangioma & 1 & & & & & \\
\hline
\end{tabular}


cipalmente em animais idosos. De acordo com Moore et al. (2001) e Bonnett et al. (2005), a alta prevalência de neoplasias deve-se ao aumento da longevidade em animais de companhia nos últimos anos, aumentando a incidência de neoplasias entre essa população. Foi observado, também, que as neoplasias constituem uma importante causa de morte em pequenos animais, correspondendo a $11 \%$ das mortes. Resultados semelhantes foram encontrados por outros autores, que relatam mortalidade por neoplasias entre $7,8 \%$ a 13,8\% do total de mortes em cães (Bentubo et al. 2007, Fighera et al. 2008, Trapp et al. 2010) e gatos (Trapp et al. 2010). A alta frequência de metástases $(20,1 \%)$ e o elevado número de tumores malignos $(78 \%$ em cães e $95,8 \%$ em gatos) justificam a alta mortalidade observada.

A incidência de tumores em fêmeas foi maior que em machos, resultado este semelhante ao obtido em diversos estudos (De Nardi et al. 2002, Pires et al. 2003, Vascellari et al. 2009), provavelmente relacionado a alta incidência de neoplasmas mamários (Pires et al. 2003, Lana et al. 2007, Merlo 2008). Em relação à idade, o resultado exposto corrobora com o descrito por De Nardi et al. (2002) e Pires et al. (2003), onde animais idosos foram mais frequentemente afetados por processos tumorais.

Em relação ao sistema afetado, os resultados obtidos são semelhantes aos mencionados por Souza et al. (2001), em Salvador e Vascellari et al. (2009), na Itália, que descrevem maior frequência de neoplasmas de pele, seguidos por neoplasmas mamários. Ao contrário, De Nardi et al. (2002) no Paraná e Pires et al. (2003) em Portugal, encontraram maior frequência de tumores mamários. Neste estudo os carcinomas de células escamosas, seguido dos hemangiossarcomas, tanto em cães quanto em gatos, foram os neoplasmas cutâneos mais prevalentes. Esses tumores estão associados, principalmente, a radiação solar (Rodaski \& Werner 2008). Na região estudada esse fato é relevante, uma vez que as temperaturas são muito altas na maior parte do ano, com dias prolongados, e grande parte dos animais, principalmente os gatos, têm acesso à rua, o que aumenta o tempo de exposição à luz solar e predispõe ao desenvolvimento desses tumores.

Deve-se ressaltar também, que os três casos de colangiocarcinomas diagnosticados em gatos são, provavelmente, induzidos por infestações persistentes de Platynosomum fastosum (Andrade et al. 2012). A associação de P. fastosum com o desenvolvimento de colangiocarcinomas foi descrita, no Brasil, em quatro gatas na década de 1980 (Santos et al. 1981), não tendo sido confirmada em publicações posteriores que descrevem infecção por P. fastosum em diferentes países e regiões (Andrade et al. 2012).

A ampla variedade de tumores que acomete cães e gatos é numerosa e, em decorrência disso, o diagnóstico e a escolha da terapia mais adequada pelo clínico veterinário pode ser muito difícil. Por isso, é recomendável o diagnóstico histológico sistemático de todas as lesões suspeitas de neoplasias. Além disso, são necessários estudos que determinem os fatores epidemiológicos dos tumores que acometem cães e gatos nas diferentes regiões, a fim de serem to- madas medidas de controle importantes para diminuir sua ocorrência e letalidade.

\section{REFERÊNCIAS}

Andrade R.L.F.S., Dantas A.F.M., Pimentel L.A., Galiza G.J.N., Carvalho F.K.L., Costa V.M.M. \& Riet-Correa F. 2012. Platynosomum fastosum induced cholangiocarcinomas in cats. Vet. Parasitol. (In publication) Doi: http:// dx.doi.org/10.1016/j.vetpar.2012.04.015

Bentubo H.D.L., Tomaz M.A., Bondan E.F. \& Lallo M.A. 2007. Expectativa de vida e causas de morte em cães na área metropolitana de São Paulo (Brasil). Ciência Rural 37(4):1021-1026.

Bonnett B.N., Egenvall A., Hedhammar A. \& Olson P. 2005. Mortality in over 350,000 insured Swedish dogs from 1995-2000. I. Breed-, gender-, ageand cause-specific rates. Acta Vet. Scand. 46:105-120.

Daleck C.R., De Nardi A.B. \& Rodaski S. 2008. Oncologia em cães e gatos. Roca, São Paulo. 612p.

De Nardi A.B., Rodaski S., Souza R.S., Costa T.A., Macedo T.R., Rodigheri S.M., Rios A. \& Piekarz C.H. 2002. Prevalência de neoplasias e modalidades de tratamento em cães atendidos no Hospital Veterinário da Universidade Federal do Paraná. Archs Vet. Sci. 7(2):15-26.

Fighera R.A., Souza T.M., Silva M.G., Brum J.S., Graça D.L., Kommers G.D., Irigoyen L.F. \& Barros C.S.L. 2008. Causas de morte e razões para eutanásia de cães da Mesorregião do Centro Ocidental Rio-Grandense (19652004). Pesq. Vet. Bras. 28(4):223-230.

Lana S.E., Rutteman G.R. \& Withrow S.J. 2007. Tumors of the mammary gland, p.619-636. In: Withrow S.J. \& Vail D.M. (Eds), Withrow and MacEwen's Small Animal Clinical Oncology. $4^{\text {th }}$ ed. Saunders Elsevier, St Louis.

Merlo D.F. 2008. Cancer incidence in pet dogs: Findings of the animal tumor registry of Genoa, Italy. J. Vet. Intern. Med. 22(4):976-984.

Meuten D.J. 2002. Tumors in Domestic Animals. $4^{\text {th }}$ ed. Iowa State Press, Ames. 788p.

Misdorp W., Else R.W., Hellmén E. \& Lipscomb T.P. 1999. International Histological Classification of Tumors of Domestic Animals. Second Series. World Health Organization, Geneva, Switzerland.

Moore G.E., Burkman K.D., Carten M.N. \& Peterson M.R. 2001. Causes of death or reasons for euthanasia in military working dogs: 927 cases (1993-1996). J. Am. Vet. Med. Assoc. 219:209-214.

Oliveira Filho J.C., Kommers G.D., Masuda E.K., Marques B.M.F.P.P., Fighera R.A. Irigoyen L.F. \& Barros C.S.L. 2010. Estudo retrospectivo de 1.647 tumores mamários em cães. Pesq. Vet. Bras. 30(2):117-185.

Pires M.A., Travassos F.S. \& Pires I. 2003. Neoplasias em canídeos: um estudo descritivo de 6 anos. Revta Port. Ciênc. Vet. 98:111-118.

Rodaski S. \& Werner J. 2008. Neoplasias de pele, p.253-279. In: Daleck C.R., De Nardi A.B. \& Rodaski S. (Eds), Oncologia em Cães e Gatos. Roca, São Paulo.

Santos J.A., Lopes M.A.F., Schott A.C., Santos A.E.S., Porfírio L.C. \& Passos L. 1981. Colangiocarcinomas em gatos com parasitismo de ductos biliares por Platynossomum fastosum. Pesq. Vet. Bras. 1:31-36.

Souza V.T.F., Paraguassu A.A. \& Moreira E.L.T. 2001. Ocorrência de neoplasias em cães na cidade de Salvador, Bahia (achados de biópsias). Revta Brasil. Saúde Prod. Anim. 2(2):53-58.

Souza T.M. 2005. Estudo retrospectivo de 761 tumores cutâneos em cães. Dissertação de Mestrado em Patologia Veterinária, Universidade Federal de Santa Maria (UFSM), Santa Maria, RS. 280p.

Trapp S.M., Iacuzio A.I., Barca Junior F.A., Kemper B., Silva L.C., Okano W., Tanaka N.M., Grecco F.C.A.R., Cunha Filho L.F.C. \& Sterza F.A.M. 2010. Causas de óbito e razões para eutanásia em uma população hospitalar de cães e gatos. Braz. J. Vet. Res. Anim. Sci. 47(5):395-402.

Vascelari M., Baioni E., Ru G., Carminato A. \& Mutinelli F. 2009. Animal tumor registry of two provinces in northern Italy: Incidence of spontaneous tumors in dogs and cats. BMC Vet. Res. 5:39. 